\title{
Parametric Amplification of Metric Fluctuations During Reheating in Two Field Models
}

\author{
F. Finelli ${ }^{1,2}$ and R. Brandenberger ${ }^{3}$ \\ ${ }^{1}$ Department of Physics, Purdue University, West Lafayette, IN 47907, USA; \\ ${ }^{2}$ Department of Physics and INFN, I-40126 Bologna, Italy; \\ ${ }^{3}$ Department of Physics, Brown University, Providence, RI 02912, USA;
}

(October 25, 2018)

\begin{abstract}
We study the parametric amplification of super-Hubble-scale scalar metric fluctuations at the end of inflation in some specific two-field models of inflation, a class of which is motivated by hybrid inflation. We demonstrate that there can indeed be a large growth of fluctuations due to parametric resonance and that this effect is not taken into account by the conventional theory of isocurvature perturbations. Scalar field interactions play a crucial role in this analysis. We discuss the conditions under which there can be nontrivial parametric resonance effects on large scales.
\end{abstract}

PACS numbers: $98.80 \mathrm{Cq}$

\section{INTRODUCTION}

It was recently suggested [1] that parametric resonance during the reheating phase of an inflationary Universe [2. 14 may lead to an exponential amplification of superHubble scale gravitational fluctuations. If true, this would affect the usual predictions of inflationary models for observables such as the matter power spectrum and the spectrum of cosmic microwave anisotropies. In particular, it would require the coupling constants in the particle physics model of inflation to be exponentially smaller than previously thought in order that the theory does not generate a too large amplitude for the fluctuations.

In Ref. [5] it was shown that, although there are no causality constraints which prohibit the amplification of super-Hubble (but sub-horizon) modes during reheating, the effect does not occur in a simple massive scalar field model of chaotic inflation based on the potential $V(\phi)=m^{2} \phi^{2} / 2$ (Here, $\phi$ is the inflaton field). This is true even beyond the linear analysis [6]. Similarly, there is no effect for a quartic potential [7] $V(\phi)=\lambda \phi^{4} / 4$, nor for a potential containing both quadratic and quartic terms 8]. These results agree with the earlier analyses in Refs. 90 and [10].

It was then suggested 11] that the amplification of super-Hubble-scale modes would occur for two field models of inflation, e.g. for a model with potential

$$
V(\phi, \chi)=\frac{1}{2} m^{2} \phi^{2}+\frac{1}{2} g^{2} \phi^{2} \chi^{2},
$$

where as before $\phi$ is the inflaton field and $\chi$ is a second scalar matter field. This model had earlier been analyzed by Taruya and Nambu [12] who claimed that the isocurvature mode of the fluctuations will be parametrically amplified during reheating. However, as was shown in Refs. [13] and [14] (and more recently in [15]), the fluctuations in the $\chi$ field are exponentially suppressed during inflation for values of the coupling constant for which the equation of motion of the metric fluctuations corresponds to broad resonance, thus rendering the effect studied in Ref. [11] completely inefficient.

The suppression of fluctuations in the $\chi$ field which renders the parametric amplification of gravitational fluctuations ineffective in the model given by (1) occurs since during inflation the induced mass $m_{\chi}$ of the $\chi$ field which is given by $m_{\chi}=g|\phi|$ is larger than the Hubble expansion parameter $H$, and hence, as can be easily seen by considering the equation of motion

$$
\ddot{\delta} \chi+3 H \dot{\delta} \chi+\left(\frac{k^{2}}{a^{2}}+g^{2} \phi^{2}\right) \delta \chi=0
$$

for the linearized fluctuation of the $\chi$ field with comoving wave number $k$ (the scale factor is denoted by $a(t)), \delta \chi$ undergoes damped oscillatory motion.

A model in which $m_{\chi}<H$ during the stage of inflation when scales of cosmological interest today exit the Hubble radius was recently studied by Bassett and Viniegra [16]. It is a two field model given by the potential

$$
V(\phi, \chi)=\frac{1}{4} \lambda \phi^{4}+\frac{1}{2} g^{2} \phi^{2} \chi^{2} .
$$

In the absence of metric fluctuations, this model was studied in detail in Ref. 17] (see also [18]), where it was shown that for values of the coupling constants satisfying

$$
g^{2} \simeq 2 \lambda
$$

long wavelength modes $(k \simeq 0)$ are in the first broad instability band of the Floquet-type equation of motion derived from (2) after field rescaling which describes the parametric resonance of matter fluctuations in an unperturbed expanding space-time. Bassett and Viniegra 16] showed that in this model the quantity $\zeta$ [19] increases exponentially during the initial stages of reheating. Note that $\zeta$ is a measure of the curvature fluctuations and is believed to be conserved on super-Hubble scales in the absence of isocurvature fluctuations (see e.g. [20] for a 
review of the theory of cosmological fluctuations). However, since the model given by (3) admits isocurvature fluctuations, a growth of $\zeta$ on super-Hubble modes is expected also in the "usual" analysis of the evolution of fluctuations in inflationary cosmology.

In this paper we take a closer look at the theory given by the potential (3). Subject to certain assumptions on initial conditions of the background field dynamics we recover results similar to Bassett and Viniegra: exponential growth of $\zeta$ during the initial stages of reheating. Furthermore, we demonstrate that this effect is not taken into account by the conventional theory of isocurvature perturbations. We then discuss some criteria which an inflationary Universe model must satisfy in order to have substantial parametric growth of $\zeta$ during reheating via scalar field interactions. We argue that these conditions are naturally satisfied in some models of hybrid inflation, and we study a couple of concrete examples in which super-Hubble-scale gravitational fluctuations grow exponentially during reheating (some other examples where exponential growth of super-Hubble-scale modes could occur are given in [21]).

\section{MASSLESS TWO FIELD MODEL RECONSIDERED}

In this section we will consider the two-field model with potential (3) in which Bassett and Viniegra [16] showed the parametric resonance of super-Hubble scale gravitational modes. This model has been studied in detail in 177 in the absence of gravitational perturbations (see also [18]).

As in our previous paper [5], we shall work in longitudinal gauge in which the metric including linearized scalar metric fluctuations takes on the form

$$
d s^{2}=(1-2 \Phi) d t^{2}-a^{2}(t)(1+2 \Psi) d x^{i} d x_{i}
$$

where the $x^{i}$ are the comoving spatial coordinates and $t$ is physical time. Since for the matter model considered the off-diagonal components of the spatial part of the energymomentum tensor vanish, the corresponding components of the Einstein equations imply [20] $\Psi=\Phi$. For the moment we will write down the equations for a general system with multiple scalar fields $(i=1, \ldots, n)$, and only at a later stage will we specialize to the specific model considered in this section.

The remaining independent equations of motion for linearized perturbations in this Einstein-Higgs system are the perturbed energy constraint and momentum constraint equations as well as the equations of motion for the Higgs field perturbations $\delta \phi_{i}$ :

$$
\begin{aligned}
-3 H \dot{\Phi} & -\left(\frac{k^{2}}{a^{2}}+3 H^{2}\right) \Phi \\
& =4 \pi G \sum_{i=1}^{n}\left[\dot{\phi}_{i} \delta \dot{\phi}_{i}-\Phi \dot{\phi}_{i}{ }^{2}+V,,_{i} \delta \phi_{i}\right],
\end{aligned}
$$

$$
\begin{aligned}
& \dot{\Phi}+H \Phi=4 \pi G \sum_{i=1}^{n} \dot{\phi}_{i} \delta \phi_{i}, \\
& \ddot{\delta} \dot{\phi}_{i}+3 H \dot{\delta} \dot{\phi}_{i}+\left[\frac{k^{2}}{a^{2}} \delta \phi_{i}+\sum_{j=1}^{n} V_{, i j} \delta \phi_{j}\right] \\
&=4 \dot{\Phi} \dot{\phi}_{i}-2 V,,_{i} \Phi,
\end{aligned}
$$

where $k$ denotes the comoving wavenumber, $V{ }_{i}$ indicates the derivative of $V$ with respect to $\phi_{i}$, and $G$ is Newton's constant.

The Sasaki-Mukhanov 22] variables for the $n$ matter fields are

$$
Q_{i}=\delta \phi_{i}+\frac{\dot{\phi}_{i}}{H} \Phi
$$

and satisfy the following system of equations:

$$
\ddot{Q}_{i}+3 H \dot{Q}_{i}+\frac{k^{2}}{a^{2}} Q_{i}+\sum_{j=1}^{n}\left[V_{, i j}-\frac{8 \pi G}{a^{3}}\left(\frac{a^{3}}{H} \dot{\phi}_{i} \dot{\phi}_{j}\right) \cdot\right] Q_{j}=0 .
$$

We will now specialize to our two field model. If the homogeneous part of the second scalar field $\chi$ vanishes (which will not be true if parametric resonance is to excite gravitational fluctuations - see later), then the inflaton $\phi$ during the initial stages of reheating (when back-reaction effects are negligible) oscillates as follows [17]

$$
\phi(\eta)=a^{-1} \phi_{0} \operatorname{cn}\left(x-x_{0}, \frac{1}{\sqrt{2}}\right),
$$

where $\phi_{0}$ is the amplitude of $\phi$ at the end of the slowrolling period, $c n$ is the Jacobi elliptic cosine function, $\eta$ is conformal time and $x=\sqrt{\lambda} \phi_{0}$ is a rescaled dimensionless conformal time coordinate. Following [17, it is insightful to rescale all the fields $f$ by the scale factor and use new fields $\tilde{f}=a f$. The equations of motion (10) then become

$$
\begin{aligned}
\tilde{Q}_{\phi}^{\prime \prime} & +\left[\kappa^{2}-\frac{a^{\prime \prime}}{a}+3 c n^{2}\left(x-x_{0}, \frac{1}{\sqrt{2}}\right)\right] \tilde{Q_{\phi}} \\
& =-2 \frac{g^{2}}{\lambda \phi_{o}} c n\left(x-x_{0}, \frac{1}{\sqrt{2}}\right) \tilde{\chi} \tilde{Q}_{\chi}+M_{\phi \phi} \tilde{Q}_{\phi}+M_{\phi \chi} \tilde{Q_{\chi}} \\
{\tilde{Q_{\chi}}}^{\prime \prime} & +\left[\kappa^{2}-\frac{a^{\prime \prime}}{a}+\frac{g^{2}}{\lambda} c n^{2}\left(x-x_{0}, \frac{1}{\sqrt{2}}\right)\right] \tilde{Q_{\chi}} \\
& =-2 \frac{g^{2}}{\lambda \phi_{o}} \operatorname{cn}\left(x-x_{0}, \frac{1}{\sqrt{2}}\right) \tilde{\chi} \tilde{Q}_{\phi}+M_{\phi \chi} \tilde{Q}_{\phi}+M_{\chi \chi} \tilde{Q_{\chi}}
\end{aligned}
$$

where, following the notation of 16 (up to a prefactor containing $a$ ), we have have used the abbreviation

$$
M_{\phi_{1} \phi_{2}}=\frac{8 \pi G}{a^{2}}\left[\frac{1}{a H}\left(\tilde{\phi}_{1}^{\prime}-\frac{a^{\prime}}{a} \tilde{\phi}_{1}\right)\left(\tilde{\phi}_{2}^{\prime}-\frac{a^{\prime}}{a} \tilde{\phi}_{2}\right)\right]^{\prime},
$$

and where 


$$
\kappa^{2}=\frac{k^{2}}{\lambda \phi_{0}^{2}} .
$$

In the model considered, the time-averaged equation of state is that of radiation and hence the scale factor is linear in $\eta$ and thus $a^{\prime \prime}=0$.

Neglecting for a moment the terms on the right hand side of the equations, both equations (12) and (13) are of the form of Lamé equations. Lamé equations in the context of reheating were first noticed in [3] and then studied in detail in [23], 18 and [17]. The coefficient in front of the $c n^{2}$ term (which in the case of $(13)$ is $g^{2} / \lambda$ ) is crucial to the resonance structure of the equation. Since we are interested in super-Hubble modes, we need to know for which values of the coefficient the mode $\kappa=0$ lies in the first instability band of the equation. This is the case for values $\mathrm{F}$

$$
1<\frac{g^{2}}{\lambda}<3
$$

This implies that resonance occurs only in the equation (13) for $\tilde{Q}_{\chi}$, and then only for values of $g^{2} / \lambda$ in the above range (or in the range corresponding to other instability bands). However, if the condition (16) is satisfied, and if the $\chi$ field is indeed excited, then parametric amplification of $\tilde{Q_{\chi}}$ is expected, and via the terms on the right hand side of (12), induced exponential growth of $\tilde{Q_{\phi}}$ should occur.

Note, however, that it is not sufficient to show that an amplification of $\tilde{Q}_{\phi}$ or $\tilde{Q}_{\chi}$ occurs in order to demonstrate that parametric resonance during reheating will have a crucial effect on the amplitude of gravitational fluctuations. Note that also in the conventional treatment of fluctuations [20] the amplitude of the $\tilde{Q}$ variable grows during the interval in which the equation of state of the background changes. As emphasized in [5], a straight forward way to check if the effect discussed here is a new effect is to consider the time evolution of the "traditional conserved quantity" $\zeta$ ( 19]) which gives a measure of the adiabatic component of the metric fluctuations. In the multi-field case, $\zeta$ is given by [12]

$$
\zeta=\frac{H}{\sum_{j} \dot{\phi}_{j}^{2}} \sum_{i} \dot{\phi}_{i} Q_{i}
$$

In the two field case, the evolution equation for $\zeta$ is 24]

\footnotetext{
* Note that this condition and the corresponding conditions in all the other examples discussed in this paper are stable against perturbative coupling constant renormalizations, the reason being that we consider $g^{2} \sim \lambda$ and $\lambda$ should be constrained by the CMB anisotropy results to be a small number $<<1$. Thus, the perturbative correction terms which are of order $g^{4}$ are much smaller than either $g^{2}$ or $\lambda$.
}

$$
\begin{aligned}
\dot{\zeta} & =-\frac{H}{\dot{H}} \frac{\nabla^{2}}{a^{2}} \Phi \\
& +\frac{H}{2}\left[\frac{Q_{\phi}}{\dot{\phi}}-\frac{Q_{\chi}}{\dot{\chi}}\right] \frac{d}{d t}\left(\frac{\dot{\phi}^{2}-\dot{\chi}^{2}}{\dot{\phi}^{2}+\dot{\chi}^{2}}\right) .
\end{aligned}
$$

In the case of a single scalar field, the second term vanishes, but the equation cannot be applied during reheating since $\dot{H}=0$ at times when $\dot{\phi}=0$.

In the single field case, the evolution equation for $\zeta$ which applies also during reheating is (for longwavelength perturbations for which the spatial gradient term can be neglected) [5]

$$
(1+w) \dot{\zeta}=0 .
$$

This shows that $\zeta$ is conserved unless $w=-1$. Unless matter is given by an oscillating scalar field (in which case $w=-1$ will occur at the turnaround points when $\dot{\phi}=$ 0 ), Eq. (19) implies that the variable $\zeta$ is conserved on scales outside the Hubble radius [19]. However, reheating corresponds to an oscillating inflaton field, in which case the conclusion that $\zeta$ is constant may break down, as discussed in [5]. Nevertheless, in the specific single field models which have been analyzed [10,9,9, 8 ] it was found that no net increase of $\zeta$ occurs during the initial stages of reheating, and a general proof of the constancy of $\zeta$ in single field models was suggested in [8].

If matter is described, more realistically, in terms of multiple scalar fields (each of which is given by a conventional action), then it appears extremely unlikely that $w=-1$ will occur at all, since at the points in time when $\dot{\phi}=0$, the other fields will not all also be at rest, and thus the net value of $w$ will be greater than -1 . Therefore, the only realistic possibility for growth of $\zeta$ is as a consequence of the second term on the right hand side of (18), a term which corresponds to an isocurvature perturbation. Inspection of (18), however, immediately shows that during reheating there is the chance of having a very large increase in $\zeta$ as a consequence of the zeros in $\dot{\phi}$ which arise periodically in time. This effect is missed if the scalar fields are treated in the slow-roll approximation, or if the change in the equation of state during reheating is modelled as a monotonic change from a nearly de-Sitter equation of state during inflation to a radiative equation of state after reheating.

However, to see if there is indeed an exponential growth of isocurvature perturbations, it is important to take a closer look at the equations. In the specific two field model of (3), symmetric initial conditions for the homogeneous part of $\chi$ give $\chi=0$ and $\dot{\chi}=0$. In this case, it follows from (17) that $\zeta$ only depends on $Q_{\phi}$, and, since by (12) there is no parametric amplification of super-Hubble modes of $Q_{\phi}$ given that the coupling to $Q_{\chi}$ vanishes, that there will therefore be no parametric amplification of $\zeta$. The same result can also be seen from (18) since for symmetric initial conditions, the time derivative on the right hand side of the equation acts 


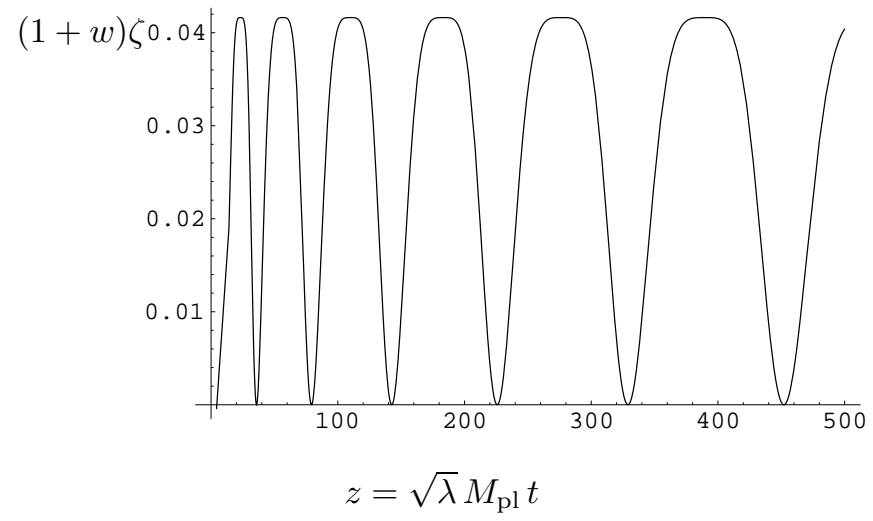

FIG. 1. Evolution of $(1+w) \zeta$ for the model of Eq. (3) as a function of the a-dimensional time $z=\sqrt{\lambda} M_{\mathrm{pl}} t$ for $\chi_{0}=\dot{\chi}_{0}=0$ and $\phi_{0}=3.5 M_{\mathrm{pl}}, \dot{\phi}_{0}=-.1 M_{\mathrm{pl}}$ as initial conditions for the background. The fluctuation $Q_{\phi}$ and $Q_{\chi}$ start in the adiabatic vacuum 40 e-foldings before inflation ends. The wavenumber is $k=10^{2}$, which corresponds to five times the Hubble radius at the beginning of the simulation. Note that the mode is far outside the Hubble radius at the end of inflation.

on a constant. The result is confirmed by our numerical analysis (see Figure 1).

However, due to quantum fluctuations we expect that the average of $\chi$ over a volume corresponding to a particular super-Hubble (but sub-horizon) mode will not vanish. It is reasonable [25,26] to use for the homogeneous value of $\chi$ the r.m.s. value of the renormalized quantum fluctuations. It follows from (13) that $Q_{\chi}$ will experience parametric amplification during the initial stages of reheating. It will grow as $\exp \left[\mu_{0} \eta\right]$, where $\mu_{0}$ is the Floquet index of $k=0$ (which for continuity reasons cannot be too different from the Floquet exponent for long wavelengths). Via the non-vanishing source terms in (12), this will induce parametric growth of $Q_{\phi}$, and this quantity mainly will contribute to the parametric growth of $\zeta$. Our numerical analysis confirms the above considerations. In Figure 2 we depict the growth of $\zeta$ during the initial stages of reheating for two different values of the homogeneous component of $\chi$, which shows how the onset of the parametric growth of $\zeta$ is dependent on the value of $\chi$. In Figure 3 we show how the growth of $\zeta$ is similar to the growth of $Q_{\phi}$.

\section{ISOCURVATURE PERTURBATIONS}

Having determined in the previous section that in the model (3) there is indeed amplification of $\zeta$ during reheating, we must now show that this effect is indeed a consequence of parametric resonance, and not just an effect due to the change in the equation of state between the inflationary era and the post-inflationary era, an effect which is already taken care of in the "usual" theory

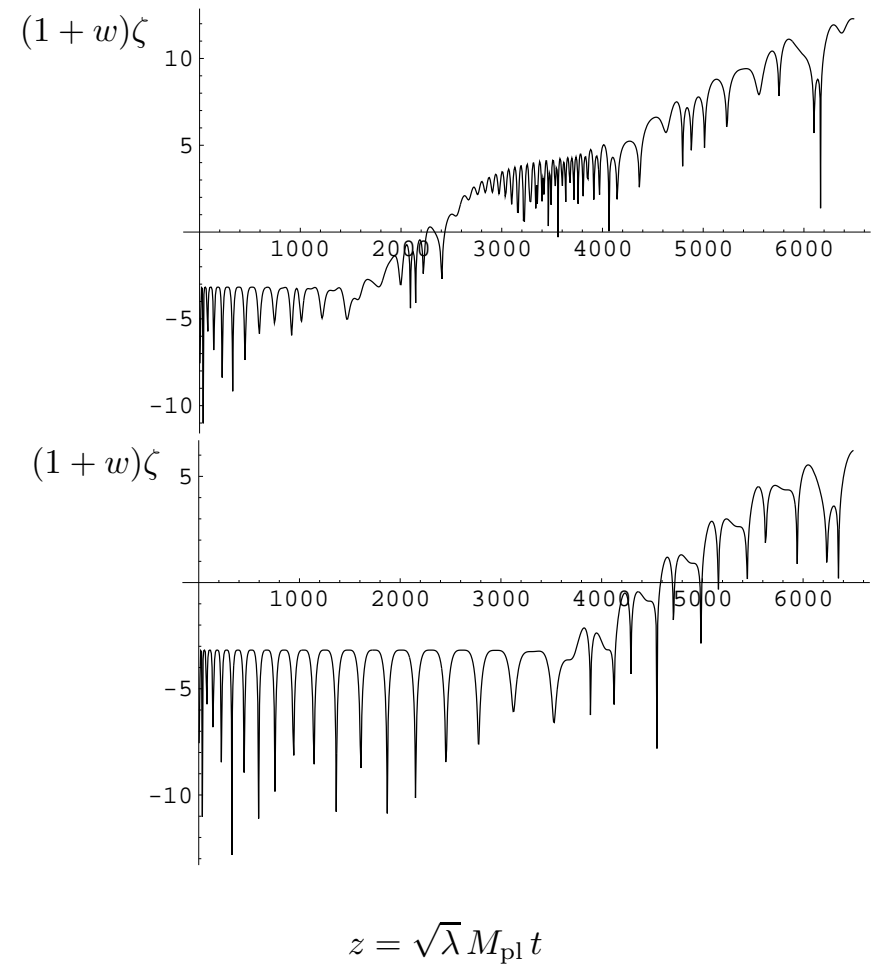

FIG. 2. Evolution in logarithmic scale of $(1+w) \zeta$ for $\chi_{0}=2 \times 10^{-2} M_{\mathrm{pl}}, \dot{\chi}_{0}=0$ (top) and $\chi_{0}=2 \times 10^{-8} M_{\mathrm{pl}}, \dot{\chi}_{0} \sim \lambda M_{\mathrm{pl}} \chi_{0}$ (bottom) as initial value for $\chi$. The initial condition for $\phi$ and $\dot{\phi}$ are the same as in Fig. 1 in both of the panels. The fluctuations $Q_{\phi}$ and $Q_{\chi}$ start in the adiabatic vacuum 40 e-foldings before inflation ends. The wavenumber is $k=10^{2}$, which corresponds to five times the Hubble radius at the beginning of the simulation. The growth of $\zeta$ is delayed in the second case because the background field, and consequently the mixing terms in Eq. (12), are smaller than in the first case: in this way $Q_{\chi}$ takes longer to feed the growth of $Q_{\phi}$ and $\zeta$. The initial conditions for the second case correspond to the values obtained through renormalization arguments. 


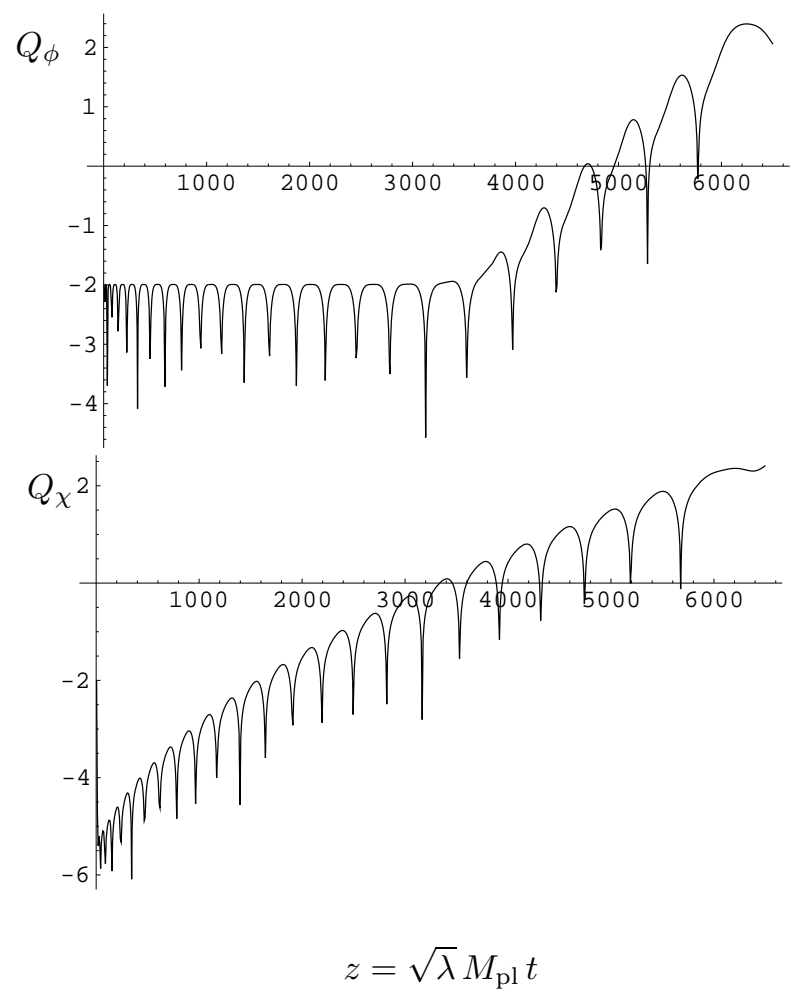

FIG. 3. Evolution in logarithmic scale of $Q_{\phi}$ (above) and $Q_{\chi}$ (below) for the second set of initial conditions of Fig. 2.

of isocurvature perturbations in inflationary cosmology, which we define as the results obtained when the transition in the equation of state between the inflationary slow-rolling phase $(p \simeq-\rho)$ and the post-inflationary radiation-dominated phase $\left(p=\frac{1}{3} \rho\right)$ is taken to be monotonic. We will show that in the "usual" analysis there can be no exponential increase in the isocurvature perturbation, and that therefore the exponential increase we obtain here is a result of parametric resonance.

The fact that isocurvature perturbations can induce an adiabatic component on super-Hubble scales has been known for a long time 27 31,20]. Entropy perturbations act as a source for $\zeta$ even on scales larger than the Hubble radius. This is true even in the case when matter is given by a single scalar field. In this case, the evolution equation for $\zeta$ becomes 32]

$$
\dot{\zeta}=3 H\left(\frac{\dot{p}}{\dot{\rho}}-\frac{\delta p}{\delta \rho}\right) .
$$

These perturbations, however, are suppressed on scales larger than the Hubble radius.

In models with two or more scalar fields, the equation for $\zeta$ is given by (18), and it is thus clear that even on super-Hubble scales one should expect $\dot{\zeta} \neq 0$. In the approximation in which both fields are slowly rolling, the time evolution of $\zeta$ on scales larger than the Hubble radius was studied in detail in 24.32,33, with particular emphasis on calculating the deviations from scale- invariance of the resulting power spectrum of density fluctuations. However, since the analyses made use of the slow-rolling approximation, no effects of the dynamics of reheating were considered.

More recently, Taruya and Nambu 12] and Bassett et al. 11] considered the effect of reheating on the spectrum of density fluctuations and discovered a large growth of $\zeta$ due to the initial isocurvature perturbations, however in a model in which the necessary initial $\chi$ field fluctuations are exponentially suppressed during inflation. Bassett and Viniegra [16] then pointed out that the suppression would be absent in the model (3).

It has been known for a long time that isocurvature perturbations can be produced in inflationary models with more than one scalar field. This issue was initially considered in the context of axion perturbations in [34], extended to more general two field models in [35] and studied in detail in [36]. It was discovered that initial super-Hubble-scale isocurvature perturbations induce an adiabatic component by the time that the scales re-enter the Hubble radius.

The gauge invariant expression for the total isocurvature perturbation in a multi-fluid system is [31]

$$
p \Gamma \equiv \sum_{i}\left(\delta p_{i}^{\mathrm{gi}}-c_{s}^{2} \delta \rho_{i}^{\mathrm{gi}}\right),
$$

where $\delta p_{i}^{\mathrm{gi}}$ and $\delta \rho_{i}^{\mathrm{gi}}$ are the gauge invariant pressure and density perturbations with respect to the total matter rest frame and the total speed of sound $c_{s}^{2}$ is defined as the weighted sum of the i-th speed of sound 31:

$$
c_{s}^{2} \equiv \frac{\dot{p}}{\dot{\rho}}=\frac{1}{\sum_{j} \dot{\phi}_{j}^{2}} \sum_{i} c_{s i}^{2} \dot{\phi}_{i}^{2}
$$

with

$$
c_{s i}^{2}=1+2 \frac{V, i}{3 H \dot{\phi}_{i}}
$$

The total isocurvature perturbation can be written as the sum of the non-adiabatic pressure component of the single component and of the relative isocurvature perturbation $S_{i j}$ as:

$$
p \Gamma=\sum_{i}\left(\delta p_{i}-c_{s i}^{2} \delta \rho_{i}\right)+\frac{1}{\sum_{i} \dot{\phi}_{i}^{2}} \sum_{i, j} \frac{\dot{\phi}_{i}^{2} \dot{\phi}_{j}^{2}}{2} S_{i j}\left(c_{s i}^{2}-c_{s j}^{2}\right)
$$

where

$$
S_{i j} \equiv \frac{\delta \rho_{i}^{\mathrm{gi}}}{\dot{\phi}_{i}^{2}}-\frac{\delta \rho_{j}^{\mathrm{gi}}}{\dot{\phi}_{j}^{2}} .
$$

The relative isocurvature perturbation $S_{i j}$ with respect to the total matter frame can be written for our two field model as 


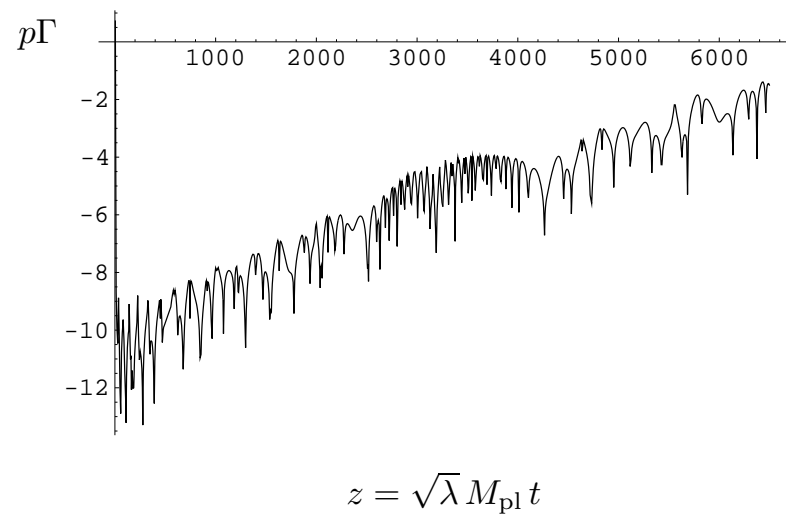

FIG. 4. Evolution in logarithmic scale of the total non-adiabatic pressure $p \Gamma$ for the first set of initial conditions of Fig. 2.

$$
\begin{aligned}
S_{\phi \chi} & =\frac{\delta \rho_{\phi}}{\dot{\phi}^{2}}-\frac{\delta \rho_{\chi}}{\dot{\chi}^{2}}-\frac{a v}{k} Q_{\phi}\left(\frac{1}{\dot{\phi}^{2}}+\frac{1}{\dot{\chi}^{2}}\right) \\
& =\frac{\delta \rho_{\phi}}{\dot{\phi}^{2}}-\frac{\delta \rho_{\chi}}{\dot{\chi}^{2}}-\frac{3 g^{2}}{8 \pi G} \frac{\phi \chi}{\dot{\phi}^{2} \dot{\chi}^{2}}(\dot{\phi} \chi-\dot{\chi} \phi) \frac{\dot{H}}{H}(\zeta-\Phi),
\end{aligned}
$$

where $v$ is the total perturbed velocity for matter, $Q_{\phi}$ is the homogeneous energy transfer to the $\phi$ component $\left(Q_{\phi}+Q_{\chi}=0\right.$ because of total energy conservation), and now $\delta \rho_{i}$ are the density perturbations in the longitudinal gauge:

$$
\delta \rho_{i}=\dot{\phi}_{i} \delta \dot{\phi}_{i}-\Phi \dot{\phi}_{i}^{2}+V,_{i} \delta \phi_{i}
$$

From Equation (26) it follows that the parametric resonance from the matter sector of the theory (the $Q$ variables to be specific) induces exponential growth of the relative isocurvature perturbation, and hence also of the total isocurvature perturbation (see Figure 4).

In turn, isocurvature perturbations determine the change in $\zeta$ via Eq. (18). This shows that in the presence of scalar field interaction terms, there is a correlated exponential growth of $\zeta$ and of the relative isocurvature perturbation $S_{\phi \chi}$. This exponential growth is a consequence of parametric resonance and is absent if the phase transition is modelled with a monotonically increasing value of $w$.

Analogously to Eq. (17) for the Bardeen parameter, the total non adiabatic pressure $p \Gamma$ can be expressed in terms of the Sasaki-Mukhanov variables in the following way:

$p \Gamma=\sum_{i}\left[\frac{\dot{V}}{\rho} \dot{\phi}_{i} Q_{i}-2 V,{ }_{i} Q_{i}-2 \frac{\dot{V}}{3 H \sum_{i} \dot{\phi}_{i}^{2}}\left(\dot{\phi}_{i} \dot{Q}_{i}+V,{ }_{i} Q_{i}\right)\right]$.

As mentioned above, the exponential growth of $S_{\phi \chi}$ and $\zeta$ during reheating is a new effect due entirely to parametric resonance. The growth of fluctuations in inflationary models with two uncoupled fields were studied in 36 in an approximation in which the oscillations of the inflaton field were neglected. In this case there is no growth of $S_{\phi \chi}$. An initial isocurvature perturbation does induce the growth of an adiabatic component on super-Hubble scales, but the final amplitude of the adiabatic mode is not much larger than the initial amplitude of the isocurvature perturbation, in agreement with the earlier analysis in 34.

\section{THREE GOLDEN RULES}

Based on the analysis of Section 2, it appears that several conditions are required in order to have efficient parametric resonance of super-Hubble-scale metric fluctuations.

1. In the absence of gravitational perturbations there must be broad-band parametric resonance in the matter sector of the theory corresponding to isocurvature fluctuations, and $k=0$ must be part of the resonance band.

2. The fluctuations in the matter field which undergoes parametric resonance must be effectively massless during inflation. More precisely, there should be no large net suppression of these fluctuations before the phase of parametric resonance.

3. The homogeneous value of the matter field which undergoes resonance must be non-vanishing. This is the weakest of the three conditions since it is only required if we work strictly to first order in perturbations.

We show how these three rules are satisfied in another model with massless fields, but now based on negative coupling instability [37. The potential is the following:

$$
V(\phi, \chi)=\frac{1}{4} \lambda \phi^{4}-\frac{1}{2} g^{2} \phi^{2} \chi^{2}+\frac{1}{4} \lambda_{\chi} \chi^{4}
$$

with the parameter $r \equiv \lambda \lambda_{\chi} / g^{4}>1$ in order to have a potential bounded from below 37]. This model has an attractor for $\chi$ in the point which minimize the potential for $\chi$

$$
\bar{\chi}(t) \sim \frac{g}{\sqrt{\lambda_{\chi}}} \phi(t) .
$$

In this way even the third and weakest of the above conditions is satisfied. The second one is easily satisfied because of the negative effective mass for the $\chi$ fluctuations when the background $\chi$ is small. In order to find the unstable bands one can use the attractor solution and estimate the frequency of the inflaton $\phi$ and of the fluctuations $\delta \chi$ during the period of coherent oscillations:

$$
\begin{gathered}
\omega_{\phi}^{2}=\lambda \phi^{2}-g^{2} \chi^{2} \sim \lambda \phi^{2}\left(1-\frac{1}{r}\right) \equiv \tilde{\lambda} \phi^{2} \\
\omega_{\delta \chi}^{2}=\frac{k^{2}}{a^{2}}+3 \lambda_{\chi} \chi^{2}-g^{2} \phi^{2} \sim \frac{k^{2}}{a^{2}}+2 g^{2} \phi^{2} .
\end{gathered}
$$

If $\chi$ is small compared to the inflaton $\phi$, then an unstable band for $k=0$ should be located at $2 g^{2}=2 \tilde{\lambda}$. This gives a second order equation for $g^{2}$ whose positive root is: 


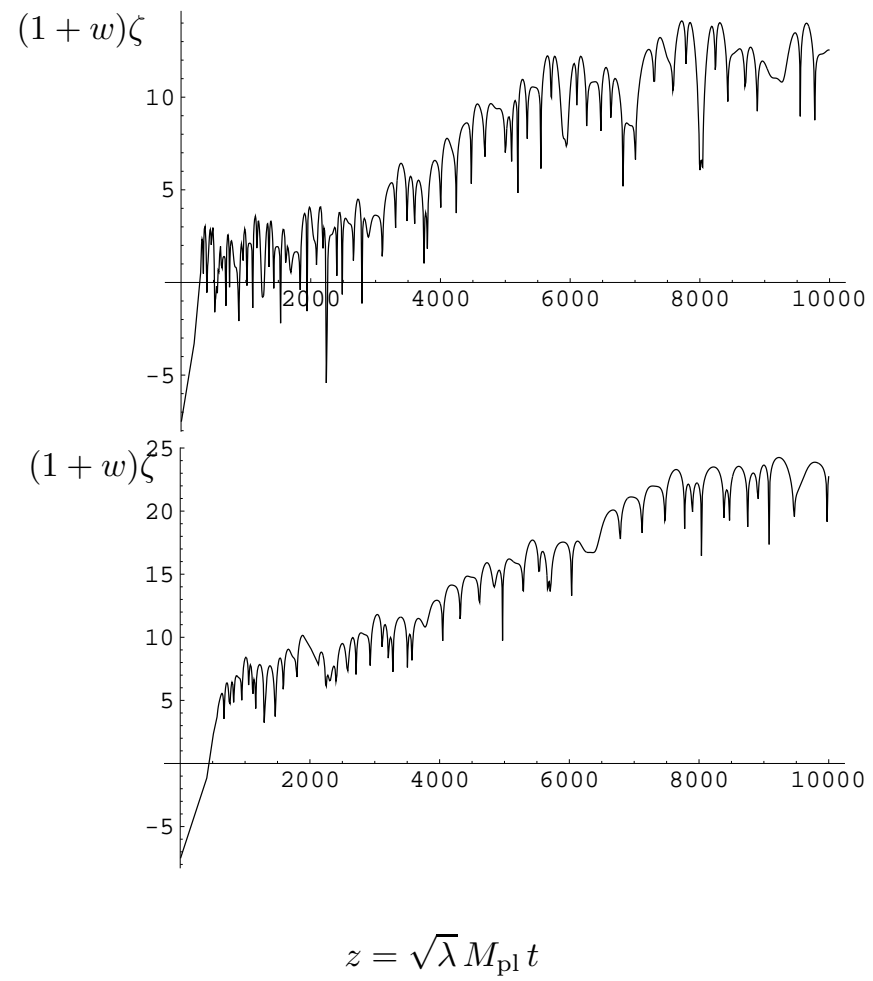

FIG. 5. Evolution in logarithmic scale of $(1+w) \zeta$ for the model (29) for $g^{2}=\lambda, \lambda_{\chi}=10^{2} \lambda$ (top) and $\lambda=\lambda_{\chi}, g^{2}=\lambda / 2(\sqrt{5}-1)$ (bottom). The initial conditions for the background are $\phi_{0}=3.5 M_{\mathrm{pl}}, \dot{\phi}_{0}=-.1 \sqrt{\lambda} M_{\mathrm{pl}}, \chi_{0}=210^{-8} \times M_{\mathrm{pl}}$ and $\dot{\chi}_{0}=0$. The fluctuations $Q_{\phi}$ and $Q_{\chi}$ start in the adiabatic vacuum 40 e-foldings before inflation ends. The wavenumber is $k=10^{2}$, which roughly corresponds to five times the Hubble radius at the beginning of the simulation.

$$
g^{2}=\lambda_{\chi} \frac{-1+\sqrt{1+4 \lambda / \lambda_{\chi}}}{2}
$$

We confirm numerically this analytical estimate in Figure 5 for two allowed values of $g^{2}: g^{2}=\lambda$ with $\lambda \ll \lambda_{\chi}$ and $g^{2}=\lambda / 2(\sqrt{5}-1)$ with $\lambda=\lambda_{\chi}$. The reason for the growth of $\zeta$ is similar to the previous case: $Q_{\chi}$ is parametrically amplified, feeds the growth of $Q_{\phi}$ and in this case both contribute to the growth of $\zeta$.

\section{A MODEL MOTIVATED BY HYBRID INFLATION}

Another natural scenario in which the above conditions can all be satisfied is hybrid inflation [38]. Hybrid inflation is also an attractive framework for implementing inflation in the context of supergravity models [39]. Since (at least) two fields are involved in the dynamics of hybrid inflation, the generation of isocurvature perturbations is rather natural. In hybrid models, the phase of inflation during which the inflaton field $\phi$ is slowly rolling towards $\phi=0$ is terminated by a phase transition in the second scalar field $\chi$, a field with the double-well potential. This implies that during the oscillations of $\phi$, the background value of $\chi$ is non-vanishing, leading to an obvious realization of condition (3) above.

Parametric resonance in the matter sector of hybrid inflation models was studied in detail by Garcia-Bellido and Linde [40], and, in supersymmetric hybrid inflation, by Bastero-Gil et al. 41. The resonance of the fluctuations of the two fields $\phi$ and $\chi$ is inefficient for a large set of the parameter space since both the $\delta \phi$ and $\delta \chi$ fields are effectively massive during the regime of coherent oscillations $(\delta \chi$ becomes massive through the Higgs mechanism). Quite generically, parametric resonance could be much more efficient if there is a third field $\psi$ which couples to both $\phi$ and $\chi$. Another interesting possibility is to consider a doublet for the field $\chi$. Then, even in the phase of coherent oscillations, there is a massless degree of freedom, namely the "Goldstone" mode. Such a situation arises naturally in supergravity models [39].

Therefore, as a toy model we will consider the following potential for the inflaton field $\phi$ and the doublet $\chi=$ $\frac{1}{\sqrt{2}}\left(\chi_{1}, \chi_{2}\right)$ :

$$
\begin{aligned}
V & =\lambda\left(\frac{M^{2}}{2 \lambda}-|\chi|^{2}\right)^{2}+\frac{1}{2} m^{2} \phi^{2}+g^{2} \phi^{2}|\chi|^{2} \\
& =\frac{1}{2} m^{2} \phi^{2}+\frac{1}{2} g^{2} \phi^{2}\left(\chi_{1}^{2}+\chi_{2}^{2}\right)+\frac{M^{4}}{4 \lambda} \\
& -\frac{1}{2} M^{2}\left(\chi_{1}^{2}+\chi_{2}^{2}\right)+\frac{\lambda}{2} \chi_{1}^{2} \chi_{2}^{2} \\
& +\frac{\lambda}{4}\left(\chi_{1}^{4}+\chi_{2}^{4}\right) .
\end{aligned}
$$

For supersymmetric hybrid inflation, there is only one independent coupling constant since

$$
\lambda=\frac{g^{2}}{2} .
$$

The values of the masses and coupling constants are constrained by the amplitude of density fluctuations at Hubble radius crossing, which is given by [38]

$$
\frac{\delta \rho}{\rho} \sim \frac{g}{\lambda^{3 / 2}}\left(\frac{M}{M_{p l}}\right)^{3}\left(\frac{M}{m}\right)^{2}
$$

which should be about $10^{-5}$. In our numerical simulations, we choose $g^{2}=10^{-3}, \lambda=g^{2} / 2$ (as in a supersymmetric model), $M^{2} / M_{p l}^{2}=10^{-12}$ and $M^{2} / m^{2}=10^{10}$. With these values, the Hubble parameter during the late stages of inflation is much larger than $m$ which ensures slow rolling of $\phi$.

In this model, slow-roll inflation takes place while the value of the inflaton $\phi$ is larger than $\phi_{c}=M / g$. For these values, the effective square mass $m_{\chi}^{2}$ of $\chi$ (evaluated at $\chi=0)$ is positive. Once $\phi$ drops below $\phi_{c}$, the $m_{\chi}^{2}$ turns negative and inflation ends (at time $t_{c}$ ) via a symmetry breaking transition in the matter sector. We choose the 
basis of the $\chi$ fields such that the order parameter of the transition is $\chi_{1}$. However, since in general the initial ratio of $\chi$ fields and $\chi$ velocities at $t_{c}$ is not the same

$$
\frac{\dot{\chi}_{2}}{\dot{\chi}_{1}}\left(t_{c}\right) \neq \frac{\chi_{2}}{\chi_{1}}\left(t_{c}\right)
$$

we can with no loss of generality assume that the value of $\chi_{2}$ at the time $t_{r}$, when the $\chi$ transition is complete and the $\phi$ oscillations start, does not vanish (as in the previous case a reasonable value for $\chi_{2}$ is the r.m..s. of the renormalized quantum fluctuations). Thus, we have argued that the third of the conditions mentioned at the beginning of this section (non-vanishing background matter fields) is naturally satisfied in this model (in contrast to the model considered in Section 2). The initial values of the matter fields at the beginning of the period of oscillation will be

$$
\begin{aligned}
\chi_{1}\left(t_{r}\right) & \simeq M / \sqrt{\lambda} \\
0 \neq \chi_{2}\left(t_{r}\right) & \ll M / \sqrt{\lambda} .
\end{aligned}
$$

In contrast to the model (11) in which the matter fluctuations are exponentially suppressed during inflation, the effective negative coupling instability in the matter fields in the time interval between $t_{c}$ and $t_{r}$ leads to the conclusion that the $\chi$ fluctuations are not suppressed. In fact, they are suppressed during slow-roll inflation, but build up again exponentially fast in the time interval $t_{c}<t<t_{r}$. To see this, we focus on the evolution of the fluctuations in of the $\chi$ field since, as we shall see below, these are essential for the effectiveness of parametric resonance. We will consider field fluctuations neglecting metric perturbations and the mixing terms deriving from particle interactions (even if in the hybrid models these mixing terms are not perturbatively small and their importance has been emphasized in 41]). Under these approximations the evolution equation for $\delta \chi_{2}$ is

$$
\ddot{\delta} \chi_{2}+3 H \dot{\delta} \chi_{2}=-\left(\frac{k^{2}}{a^{2}}+g^{2} \phi^{2}+\lambda \chi_{1}^{2}+3 \lambda \chi_{2}^{2}-M^{2}\right) \delta \chi_{2}
$$

For $\phi>\phi_{c}$, the effective square mass is larger than $H^{2}$ and positive, thus leading to damped oscillatory solutions

$$
\delta \chi_{2} \sim a^{-3 / 2}(t) \exp (i \omega t)
$$

with $\omega=g \phi$ (in the adiabatic limit). During this time interval, however, the homogeneous components of the matter fields are also damped. The evolution equation for the order parameter $\chi_{1}$ is

$$
\ddot{\chi}_{1}+3 H \dot{\chi}_{1}=-\left(g^{2} \phi^{2}+\lambda \chi_{1}^{2}+\lambda \chi_{2}^{2}-M^{2}\right) \chi_{1}
$$

Since (up to the contributions from $\chi_{2}^{2}$ which are negligible during inflation) the effective masses in (39) and (37) are the same, the damping rates of $\chi_{1}$ and $\delta \chi_{2}$ are also the same for $t<t_{c}$. In the time interval between $t_{c}$ and $t_{r}$, the signs of the effective square masses in both Equation (39) for $\chi_{1}$ and Equation (37) for $\delta \chi_{2}$ are reversed. In both cases, the effective $m^{2}$ is now $-M^{2}$, leading to exponential increase in both $\chi_{1}$ and $\delta \chi_{2}$. This period ends when $\chi_{1}$ reaches the minimum of the potential at time $t_{r}$. To summarize the above discussion, the evolution of $\chi_{1}$ follows

$$
\begin{aligned}
\frac{M}{\sqrt{\lambda}} \simeq \chi_{1}\left(t_{r}\right) & \sim e^{M\left(t_{r}-t_{c}\right)} \chi_{1}\left(t_{c}\right) \\
& \sim e^{M\left(t_{r}-t_{c}\right)} e^{-\frac{3}{2} H\left(t_{c}-t_{i}\right)} \chi_{1}\left(t_{i}\right)
\end{aligned}
$$

where $t_{i}$ is the time at the beginning of inflation and $H$ is the Hubble constant during inflation, assumed to be constant to make the equation simple (this assumption does not affect the basic point we are making). In comparison, the evolution of $\delta \chi_{2}$ obeys

$$
\begin{aligned}
\delta \chi_{2}\left(t_{r}\right) & \sim e^{M\left(t_{r}-t_{c}\right)} \delta \chi_{2}\left(t_{c}\right) \\
& \sim e^{M\left(t_{r}-t_{c}\right)} e^{-\frac{3}{2} H\left(t_{c}-t_{i}\right)} \delta \chi_{2}\left(t_{i}\right)
\end{aligned}
$$

which shows that the exponential growth of $\delta \chi_{2}$ for $t_{c}<t<t_{r}$ precisely makes up for the exponential decay during the period $t_{1}<t<t_{c}$, exactly as it does for the evolution of $\chi_{1}$. Equations (40) and (41) can be combined to give

$$
\delta \chi_{2}\left(t_{r}\right) \sim \frac{M / \sqrt{\lambda}}{\chi_{1}\left(t_{i}\right)} \delta \chi_{2}\left(t_{i}\right)
$$

This demonstrates that there is no overall suppression of the fluctuations in $\delta \chi_{2}$ before the onset of parametric resonance, showing that the second condition for the effectiveness of parametric amplification of super-Hubble gravitational modes mentioned at the beginning of this section is satisfied.

The final conditions to discuss are the criteria for parametric resonance of the $k=0$ modes of the matter perturbations. To do this, we consider the mode equation for $\delta \chi_{2}$ during the period in which the inflaton $\phi$ oscillates. For general hybrid models, the background dynamics is chaotic since both $\phi$ and $\chi_{1}$ oscillate with different frequencies. However, in the supersymmetric case [41], the frequencies coincide and the background dynamics becomes non-chaotic. Both $\phi$ and $\chi_{1}$ oscillate with the frequency $\sqrt{2} M$. To simplify the analysis, we shall neglect the back-reaction of particle production and expansion on the inflaton, and neglect the Hubble damping term in the equation of motion (this is a good approximation since we are considering a case in which $H<<\sqrt{2} M$ and the fields oscillation are not damped by the expansion of the universe). Therefore, we take the inflaton to oscillate with amplitude $\phi_{a}<\phi_{c}$, and $\chi_{1}$ will oscillate about its ground state as

$$
\chi_{1}(z)=\frac{M}{\sqrt{\lambda}}(1+f(z))
$$


where $f(z)$ is periodic with period $2 \pi$. It is convenient to introduce the dimensionless time $z=\sqrt{2} M t$. Denoting the derivative with respect to $z$ by a prime, the equation for the Fourier mode $\chi_{2 k}$ of $\delta \chi_{2}$ becomes

$\chi_{2 k}^{\prime \prime}+\chi_{2 k}\left(\frac{k^{2}}{2 a^{2} M^{2}}+\frac{g^{2} \phi_{a}^{2}}{4 M^{2}}+\frac{g^{2} \phi_{a}^{2}}{4 M^{2}} \cos (2 z)+f+\frac{f^{2}}{2}\right)=0$

where we have neglected the terms in $\chi_{2}$. In the absence of the final term (the term containing $f(z)$ ), this has the form of the Mathieu equation

$$
\chi_{2 k}^{\prime \prime}+\chi_{2 k}(A(k)-2 q \cos (2 z))=0 .
$$

The value of $q$ is $q \leq 1 / 8$, the maximal value being taken on if $\phi_{a}=\phi_{c}$, and for long wavelengths $A(k) \simeq 2 q$. As can be seen from the Floquet instability charts (see e.g. Fig. 1 in [37]), these values do not correspond to efficient resonance. From the evolution of the background fields obtained from the full numerical solution of the background field equations (see Figure 6) it follows that the amplitude of oscillation $\phi_{a}$ is in fact substantially smaller than $\phi_{c}$. In contrast, $\chi_{1}$ oscillates with a large amplitude. Hence, the term containing $f(z)$ in Equation (44) is more important. This term leads to negative coupling instability (see 37 for a general discussion of resonant particle production by negative coupling instability) for small values of $k$. Hence, we expect parametric amplification of long wavelength gravitational fluctuations in our model.

The above considerations are supported by our numerical results. In Figure 6 we show the evolution of the background fields $\phi, \chi_{1}, \chi_{2}$ and $H$ as a function of time in a simulation with parameters mentioned after Equation (34), and with initial conditions $\phi_{0}=3 M_{\mathrm{pl}}, \chi_{1}=.01 M_{\mathrm{pl}}$, $\chi_{2}=.0001 M_{\mathrm{pl}}$.

As is evident, following an initial transient period the three scalar fields oscillate with the same frequency. The results for the fluctuation variables $Q_{\phi}, Q_{\chi_{1}}, Q_{\chi_{2}}$ and $\zeta$ are shown in Figure 7. The initial perturbation amplitudes were chosen to be $Q_{\phi}\left(t_{0}\right)=1, Q_{\chi_{1}}\left(t_{0}\right)=Q_{\chi_{1}}\left(t_{0}\right)=$ $10^{-4}$, and all their derivatives set to zero for a wavelength outside the Hubble radius $(k=0)$. As is evident, after the initial transient period, all four quantities grow almost with the same Floquet exponent, as expected from our analytical analysis.

At this point, an obvious question is whether the field 0.0001925 $\chi_{2}$ is essential in order to obtain parametric resonance of super-Hubble-scale cosmological fluctuations. In fact, the field fluctuations $\delta \chi_{1}$ also will experience an effective negative coupling instability [41], and therefore the presence of $\chi_{2}$ is not essential for this supersymmetric choice of the parameters. The equation of motion for $\delta \chi_{1}$ for a hybrid model with two field is

$$
\ddot{\delta} \chi_{1}+3 H \dot{\delta} \chi_{1}=-\left(\frac{k^{2}}{a^{2}}+g^{2} \phi^{2}+3 \lambda \chi_{1}^{2}-M^{2}\right) \delta \chi_{1} .
$$

The effective squared mass is large and positive during slow-rolling. At the beginning of the transient period
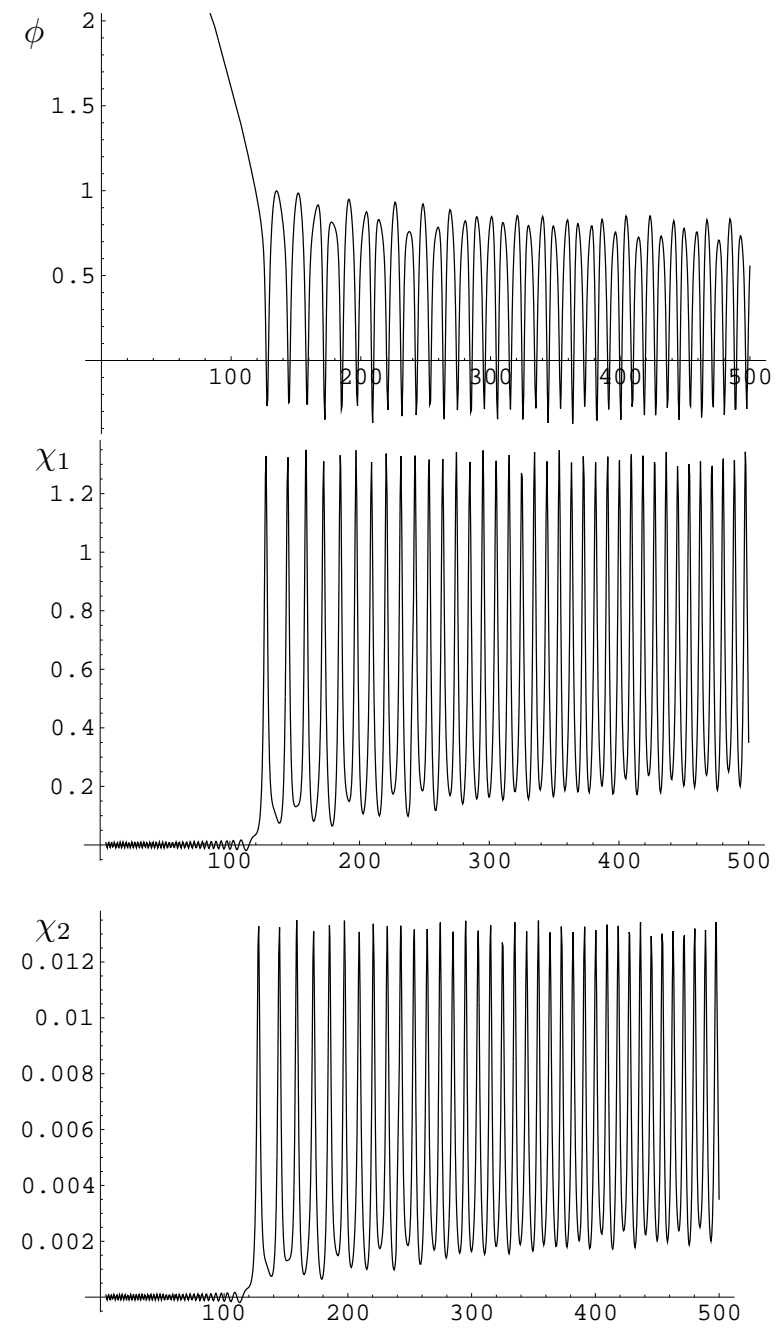

\section{H}

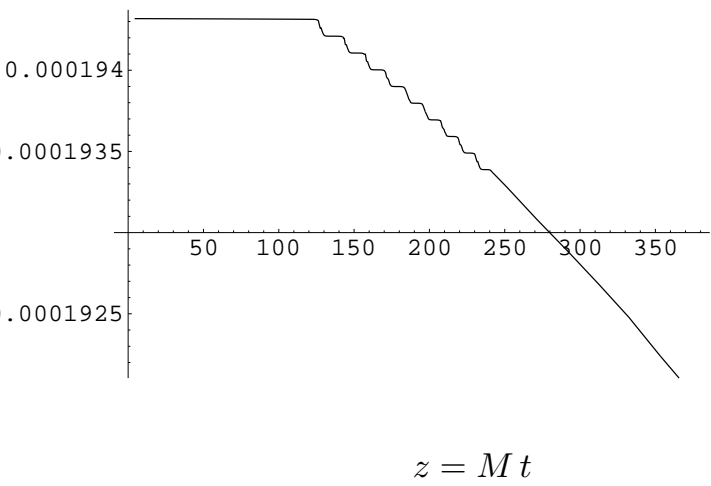

FIG. 6. Evolution of the background dynamics for a supersymmetric model of hybrid inflation with $g^{2}=10^{-3}$, $M^{2} / M_{p l}^{2}=10^{-12}$ and $M^{2} / m^{2}=10^{10}$. The initial conditions are $\phi_{0}=3 M_{\mathrm{pl}}, \chi_{1}=.01 M_{\mathrm{pl}}, \chi_{2}=.0001 M_{\mathrm{pl}}$ and all the field velocities set to zero. 

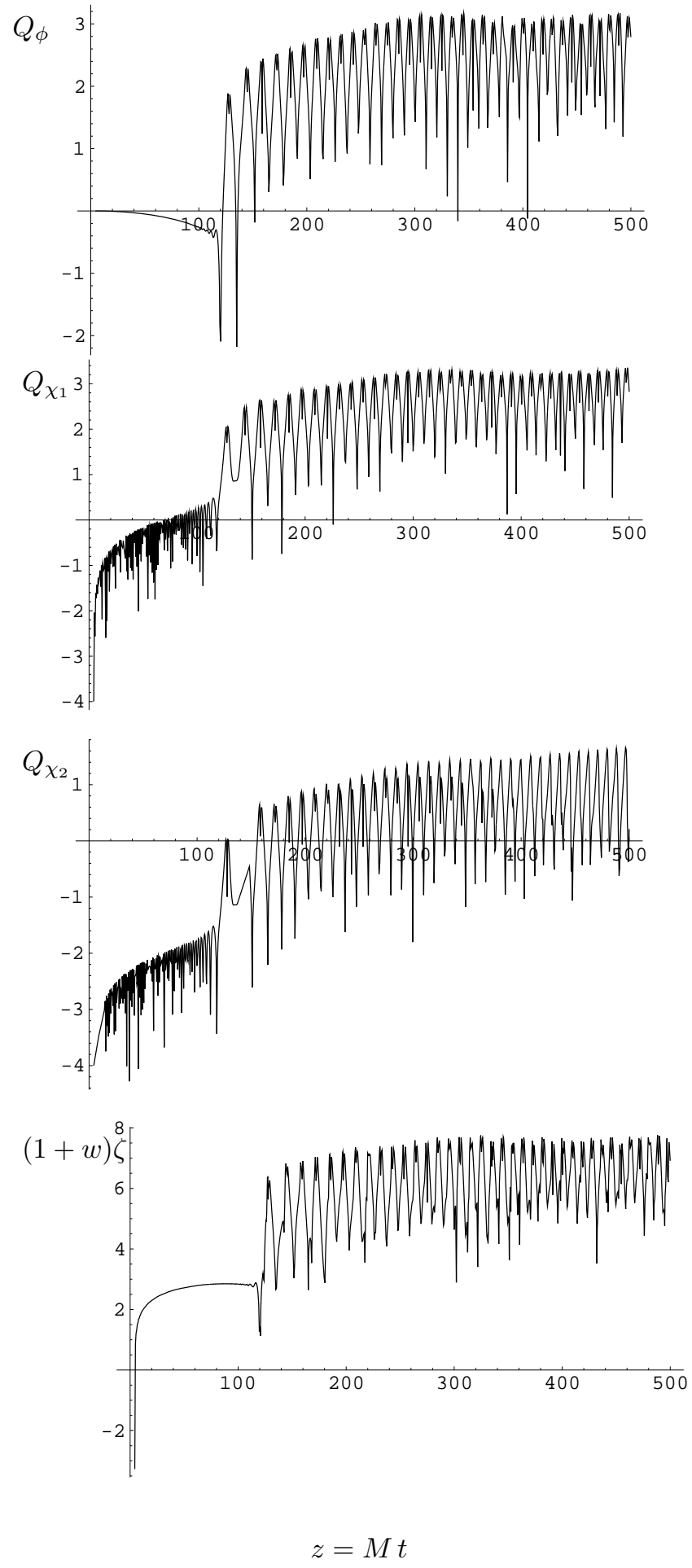

FIG. 7. Evolution in logarithmic scale for the fluctuation variables $Q_{\phi}, Q_{\chi_{1}}, Q_{\chi_{2}}$ and $(1+w) \zeta$. The initial perturbation amplitudes were chosen to be $Q_{\phi}\left(t_{0}\right)=1$, $Q_{\chi_{1}}\left(t_{0}\right)=Q_{\chi_{1}}\left(t_{0}\right)=10^{-4} M_{\mathrm{pl}}$, and all their derivatives set to zero for a wavelength outside the Hubble radius $(k=0)$. (when $\chi_{1}$ starts rolling down its potential but is not yet close to the minimum of the potential) the effective squared mass turns negative. Once $\chi_{1}$ gets close to its equilibrium position, the effective squared mass will again be large and positive (the factor 3 in the third term on the r.h.s. of (46) is crucial). However, since $\chi_{1}$ is oscillating with a large amplitude, the effect of the large mass will be periodically cancelled out by these oscillations. Neglecting the expansion of the background, Eq. (46) can be written as

$$
\chi_{1 k}^{\prime \prime}+\chi_{1 k}\left(\frac{k^{2}}{2 a^{2} M^{2}}+\frac{g^{2} \phi^{2}}{2 M^{2}}+1+3 f(z)+\frac{3}{2} f^{2}\right)=0 .
$$

From our numerical results (Figure 6) we expect that the amplitude of $f(z)$ will be only slightly smaller than 1. Hence, we expect negative coupling instability for long wavelength metric perturbations also in the two field case 41]. However, as demonstrated below for values of the coupling constants which do not correspond to the supersymmetric point, the Floquet index in the two field case will be smaller than in the three field model.

Other interesting effects happen if we go away from the supersymmetric point $g^{2}=2 \lambda$. In analogy with the results of 43. which show that random noise in the inflaton leads to an increase in the strength of the parametric instability, we expect that the chaotic background dynamics will not eliminate but rather strengthen the resonance. Chaotic background dynamics is expected for $g^{2} \sim \lambda$ in hybrid models [40]. This effect is shown by our numerical simulations (Figure 8 and Figure 9) which show that the parametric resonance of super-Hubble-scale gravitational fluctuations for the choice $g^{2}=\lambda$ is larger than in the supersymmetric case, where no chaoticity is present [41]. Figure 10 shows how the presence of the "Goldstone" mode $\chi_{2}$ changes the development of the resonance in this chaotic case. In the two field case the Floquet index with which $\zeta$ grows is smaller than the corresponding index in the three field case.

\section{DISCUSSION}

We have studied the parametric amplification of long wavelength gravitational fluctuations during reheating in two field inflationary Universe models. We have partially confirmed the results of Bassett and Viniegra 16 and shown that this effect is possible for certain models. We have established criteria under which an exponential increase in the amplitude of cosmological perturbations during the period when the inflaton field oscillates should be expected. It is crucial that there must be either broadband parametric instability or negative coupling instability in the matter sector of the theory (i.e., in the absence of gravitational perturbations). This will excite isocurvature fluctuations during reheating. It is important that there be no net exponential damping in the amplitude of 

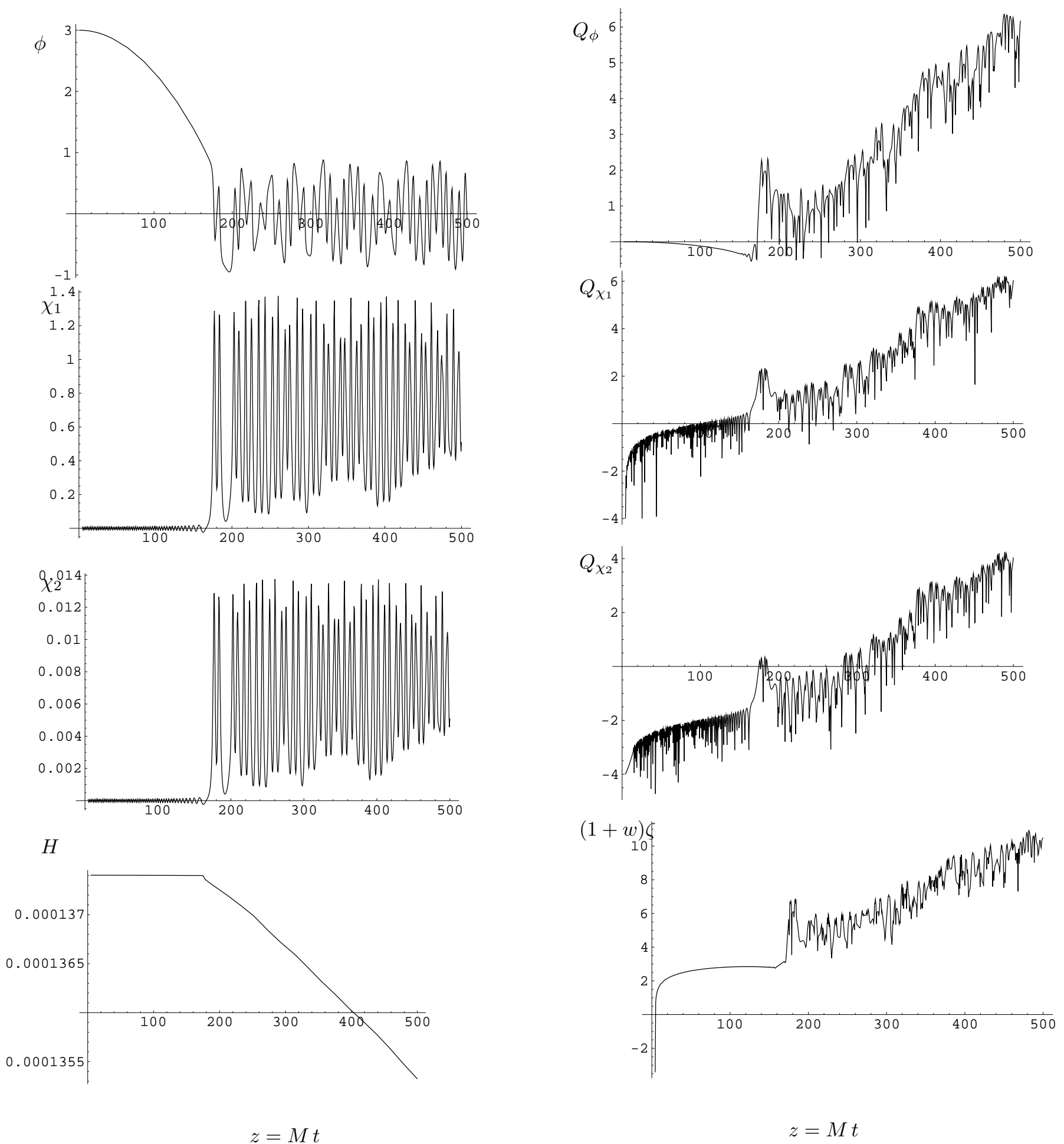

FIG. 8. Evolution of the background dynamics for a model of hybrid inflation with $g^{2}=\lambda$. The parameters and the initial conditions for the fields are the same of Fig. 6. 


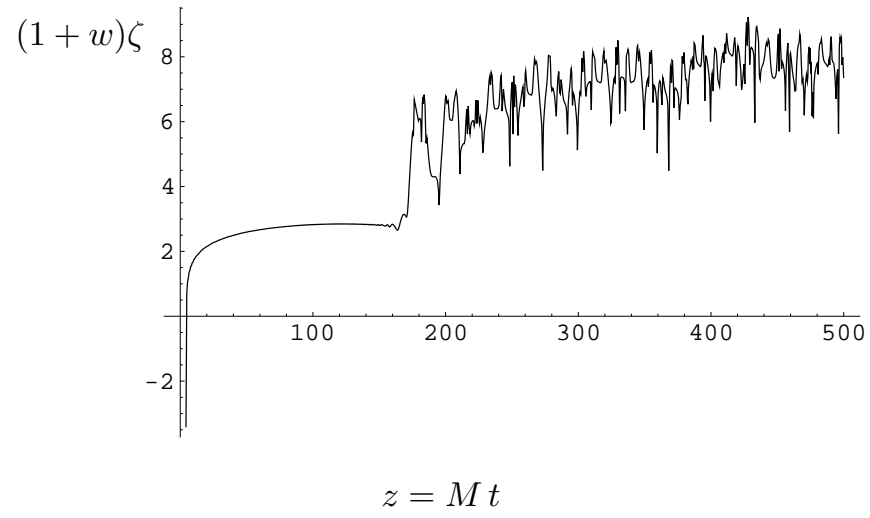

FIG. 10. Evolution in logarithmic scale of $(1+w) \zeta$ for the choice $g^{2}=\lambda$ for a two field hybrid model $\left(\chi_{2}=\delta \chi_{2}=0\right)$. The parameters, initial conditions for the background and for the perturbation amplitudes are the same of Figs. 8 and 9.

the isocurvature fluctuations before reheating. The resonance in the matter sector then induces a resonance in the gravitational sector provided that the background values of the matter fields do not vanish. Since large coupling constants are not necessary in order to have efficient resonance, the effect is stable against perturbative coupling constant renormalizations. We have shown that in this case the resulting increase in the amplitude of the adiabatic mode, conveniently tracked in terms of the variable $\zeta$, and of isocurvature fluctuations, tracked in terms of the non adiabatic pressure $p \Gamma$, is exponential and is due to the oscillations in the inflaton field. This means that the effect is absent if the phase transition is modelled by a monotonic change in $w=p / \rho$.

We then argue that the conditions under which parametric amplification of long wavelength gravitational fluctuations occurs are naturally satisfied in a class of models of hybrid inflation. The presence of a complex matter scalar field enhances the resonance, since it ensures the existence of a field which is massless in the true vacuum of the theory, but it is not crucial if there is negative coupling instability. However, note that the existence of massless modes is helpful for the effect to occur. Such massless modes arise quite generically in string theory (see e.g. 42 for a recent review). Thus, the parametric amplification of long wavelength fluctuations may be also present in models of inflation based on string theory.

\section{Acknowledgements}

We are grateful to Bruce Bassett, Serguei Khlebnikov, Lev Kofman and Bill Unruh for stimulating discussions, and Jim Zibin for comments on the draft. R.B. wishes to thank Bill Unruh for hospitality at the University of British Columbia where this work was completed. F. F. wishes to thank Brown University for hospitality. The research was supported in part (at Purdue) by the U.S. Department of Energy under Contract DE-FG0291ER40681, TASK B, and (at Brown) by DE-FG02-
91ER40688, TASK A.

[1] B. Bassett, D. Kaiser and R. Maartens, Phys. Lett. B455, 84 (1999), hep-ph/9808404.

[2] J. Traschen and R. Brandenberger, Phys. Rev. D42, 2491 (1990);

A. Dolgov and D. Kirilova, Sov. J. Nucl. Phys. 51, 172 (1990).

[3] L. Kofman, A. Linde and A. Starobinsky, Phys. Rev. Lett. 73, 3195 (1994), hep-th/9405187;

L. Kofman, A. Linde and A. Starobinsky, Phys. Rev. D56, 3258 (1997), hep-ph/9704452 (1997).

[4] Y. Shtanov, J. Traschen and R. Brandenberger, Phys. Rev. D51, 5438 (1995), hep-ph/9407247.

[5] F. Finelli and R. Brandenberger, Phys. Rev. Lett. 82, 1362 (1999), hep-ph/9809490.

[6] M. Parry and R. Easther, Phys. Rev. D59, 061301 (1999), hep-ph/9809574.

[7] R. Easther and M. Parry, hep-ph/9910441

[8] W. Lin, X. Meng and X. Zhang, hep-ph/9912510.

[9] Y. Nambu and A. Taruya, Prog. Theor. Phys. 97, 83 (1997), gr-qc/9609029.

[10] H. Kodama and T. Hamazaki, Prog. Theor. Phys. 96, 949 (1996), gr-qc/9608022;

T. Hamazaki and H. Kodama, Prog. Theor. Phys. 96, 1123 (1996), gr-qc/9609036.

[11] B. Bassett, F. Tamburini, D. Kaiser and R. Maartens, Nucl. Phys. B561, 188 (1999), hep-ph/9901319.

[12] A. Taruya and Y. Nambu, Phys. Lett. B428, 37 (1998), gr-qc/97009035.

[13] K. Jedamzik and G. Sigl, Phys. Rev. D91, 023519 (2000), hep-ph/9906287.

[14] P. Ivanov, Phys. Rev. D61, 023505 (2000), astro$\mathrm{ph} / 9906415$.

[15] A. Liddle, D. Lyth, K. Malik and D. Wands, hep$\mathrm{ph} / 9912473$.

[16] B. Bassett and F. Viniegra, hep-ph/9909353.

[17] P. Greene, L. Kofman, A. Linde, and A. Starobinsky, Phys. Rev. D56, 6175 (1997).

[18] D. Kaiser, Phys. Rev. D56, 706 (1997);

D. Kaiser, Phys. Rev. D57, 702 (1998).

[19] J. Bardeen, P. Steinhardt and M. Turner, Phys. Rev. D28, 679 (1983);

R. Brandenberger and R. Kahn, Phys. Rev. D29, 2172 (1984);

D. Lyth, Phys. Rev. D31, 1792 (1985).

[20] V. Mukhanov, H. Feldman and R. Brandenberger, Phys. Rep. 215, 203 (1992).

[21] B. Bassett, C. Gordon, R. Maartens and D. Kaiser, Phys. Rev. D61, 061302 (2000), hep-ph/9909482.

[22] M. Sasaki, Prog. Theor. Phys. 76, 1036 (1986); V. Mukhanov, JETP 67, 1297 (1988).

[23] D. Boyanovsky et al., Phys. Rev. D54, 7570 (1996).

[24] J. Garcia-Bellido and D. Wands, Phys. Rev. D52, 6739 (1995). 
[25] A. Linde, Phys. Lett. B116, 335 (1982).

[26] L. Ford and A. Vilenkin, Phys. Rev. D26, 1231 (1982).

[27] W. Press and E. Vishniac, Astrophys. J. 239, 1 (1980).

[28] J. Bardeen, Phys. Rev. D22, 1882 (1980).

[29] L. Abbott and M. Wise, Nucl. Phys. B244, 541 (1984).

[30] F. Lucchin and S. Matarrese, Phys. Lett. B164, 282 (1985); F. Lucchin and S. Matarrese, Phys. Rev. D32, 1316 (1988).

[31] H. Kodama and M. Sasaki, Prog. Theor. Phys. Suppl. 78, 1 (1984)

[32] J. Garcia-Bellido and D. Wands, Phys. Rev. D53, 5437 (1996).

[33] M. Sasaki and E. Stewart, Prog. Theor. Phys. 95, 71 (1996).

[34] M. Axenides, R. Brandenberger and M. Turner, Phys. Lett. B126, 178 (1983).

[35] A. Linde, Phys. Lett. B158, 375 (1985).

[36] S. Mollerach, Phys. Rev. D42, 313 (1990).

[37] B. Greene, T. Prokopec and T. Roos, Phys. Rev. D56, 6484 (1997), hep-ph/9705357.

[38] A. Linde, Phys. Lett. B259, 38 (1991); A. Linde, Phys. Rev. D49, 748 (1994).

[39] E. Copeland, A. Liddle, D. Lyth, E. Stewart and D. Wands, Phys. Rev. D49, 6410 (1994);

G. Dvali, Q. Shafi and R. Schaefer, Phys. Rev. Lett. 73, 1886 (1994);

G. Lazarides, R. Schaefer and Q. Shafi, Phys. Rev. D56, 1324 (1997);

G. Dvali, G. Lazarides and Q. Shafi, Phys. Lett. D424, 259 (1998), hep-ph/9710314;

A. Linde and A. Riotto, Phys. Rev. D56, 1840 (1997).

[40] J. Garcia-Bellido and A. Linde, Phys. Rev. D57, 6075 (1998), hep-ph/9711360.

[41] M. Bastero-Gil, S. King and J. Sanderson, Phys. Rev. D60, 103517 (1999).

[42] M. Dine, hep-th/0001157.

[43] V. Zanchin, A. Maia Jr., W. Craig and R. Brandenberger, Phys. Rev. D57, 4651 (1998), hep-ph/9709273; Phys. Rev. D60, 023505 (1999), hep-ph/9901207. 